\title{
Acknowledgement to Reviewers of Fermentation in 2018
}

Fermentation Editorial Office

MDPI, St. Alban-Anlage 66, 4052 Basel, Switzerland

Published: 8 January 2019

Rigorous peer-review is the corner-stone of high-quality academic publishing. The editorial team greatly appreciates the reviewers who contributed their knowledge and expertise to the journal's editorial process over the past 12 months. In 2018, a total of 103 papers were published in the journal, with a median time to first decision of 17 days and a median time to publication of 34 days. The editors would like to express their sincere gratitude to the following reviewers for their cooperation and dedication in 2018:

Acree, William E., Jr.

Adewale, Peter

Alexandri, Maria

Alfonzo, Antonio

Aoki, Dan

Aranda, Agustín

Aryal, Nabin

Bahrim, Gabriela

Balcerek, Maria

Barriuso, Jorge

Bartocci, Pietro

Benito, Santiago

Berkes, Charlotte

Berlowska, Joanna

Bernal, Vicente

Bisson, Linda F.

Bizukojc, Marcin

Bochman, Matthew L.

Bohutskyi, Pavlo

Bolton, Kim

Bubici, Giovanni

Budroni, Marilena

Campana, Raffaella

Cañete, Eduardo

Canonico, Laura

Capozzi, Vittorio

Casassa, Luis Federico

Chen, Qi

Cheng, Kuan-Chen

Cheong, Yuen Ki

Chorianopoulos, Nikos

Ciani, Maurizio
Claassens, Nico J.

Coelho, Christian

Comitini, Francesca

Congestri, Roberta

Connors, Neal

Contreras, Angela

Cordero-Bueso, Gustavo Adolfo

Cornet, Iris

Costa, Stefania

Coulibaly, Wahauwouele Hermann

Czyżowska, Agata

Dabros, Michal

Davies, Nigel

Del Carmen Portillo, Maria

Del Caro, Alessandra

Deng, Peng

Di Gioia, Diana

Dos Santos, Rui Galhano

Durall, Daniel M.

Dziekońska-Kubczak, Urszula

Edwards, Charles

Englezos, Vasileios

Escudero, Ana

Escudero, Nuria

Farber, Matthew

Ferreira, Jorge

Ferrer-Gallego, Raúl

Filannino, Pasquale

Fischer, Susann

Flythe, Michael

Fuster-Parra, Pilar

Galán, Miguel Ladero 
Gallo, Carmela

Giacosa, Simone

Giménez-Gómez, Pablo

Goncalves, Olivier

Granchi, Lisa

Guan, Wenjian

Guerrini, Simona

Gumienna, Małgorzata

Han, Kun-jun

Hogg, Timothy Alun

Hyakutake, Manami

Ibrahim, Salam A.

Jin, Zhao

Kalanetra, Karen

Kamzolova, Svetlana

Kawamura, Akira

Kim, Daehwan

Kiparissides, Costas

Kitagaki, Hiroshi

Klassen, Viktor

Koirala, Niranjan

Kordialik-Bogacka, Edyta

Kourkoutas, Yiannis

Kregiel, Dorota

Kroukamp, Heinrich

Krstanović, Vinko

$\mathrm{Ku}$, Seockmo

Kubota, Keiichi

Kucharska, Karolina

Kuno, Toshiya

Lalas, Stavros

Lee, Jechan

Lentz, Michael R.

Li, Yang

Liu, Rongming

Liu, Yuan-Shuai

Loira, Iris

Loman, Abdullah

Lopez-Moya, Federico

Love, John

Lucarini, Massimo

Lucas, Cândida

Ma, Chao

Mahboubi, Amir

Maicas, Sergi

Malfeito Ferreira, Manuel

Martorana, Alessandra

Mastanjević, Kristina

Mateusz, Mateusz

Matoulková, Dagmar

Matsumoto, Ken'ichiro

Michel, Maximilian
Morata, Antonio

Moreno, Antonio D.

Nanjundaswamy, Ananda

Nardi, Tiziana

Nieminen, Kaarlo

Nwaiwu, Ogueri

Pachapur, Vinayak

Panico, Antonio

Papapostolou, Harris

Parisi, Salvatore

Patsios, Sotiris I.

Pereira, J. A.

Pereira, Vanda

Perin, Giorgio

Pielech-Przybylska, Katarzyna

Plácido-Escobar, Jersson E.

Pretorius, Sakkie

Rahman, Khondokar Mizanur

Rakicka, Magdalena

Ranadheera, Senaka

Ray, Partha

Rinaldi, Alessandra

Rodziewicz, Joanna

Rolinec, Michal

Romano, Giovanna

Romano, Patrizia

Roubík, Hynek

Rozes, Nicolas

Russo, Pasquale

Sanmartin, Chiara

Santos, Antonio I.

Scarafoni, Alessio

Schulz, Benjamin L.

Schulz, Margot

Schweizer, Michael

Segade, Luisa

Shi, Suan

Simpson, T. J.

Snyder, Abigail

Sommer, Stephan

Souza Filho, Pedro F.

Spano, Giuseppe

Stasiak-Różańska, Lidia

Suleria, Hafiz

Tachibana, Shinjiro

Takase, Hideki

Talon, Régine

Tanimura, Ayumi

Tanner, Ralph

Thibault, Jules

Tofalo, Rosanna

Topakas, Evangelos 
Trif, Monica

Tsaltas, Dimitrios

Tufarelli, Vincenzo

Van Wyk, Niel

Varelas, Vassileios

Verardi, Alessandra

Vilela, Alice

Vodnar, Dan Cristian

Von Wright, Atte

Wahlen, Brad

Wang, Zhe

Weber, Fabian

Wenland, Juergen
Woodley, Alan

Wöstemeyer, Johannes

Wu, Sheng-Chi

Xavier, Ana Maria Rebelo Barreto

Ximenes, Eduardo

Yin, Xiang

Zago, Miriam

Zara, Giacomo

Zara, Severino

Zhang, Pangzhen

Zhong, Tuhua

Zinn, Manfred

(C) 2019 by the authors. Licensee MDPI, Basel, Switzerland. This article is an open access article distributed under the terms and conditions of the Creative Commons Attribution (CC BY) license (http://creativecommons.org/licenses/by/4.0/). 